\title{
LOW-INTENSITY ORAL ANTICOAGULATION PLUS LOW-DOSE ASPIRIN VERSUS HIGH- INTENSITY ORAL ANTICOAGULATION ALONE: A RANDOMIZED TRIAL IN PATIENTS WITH MECHANICAL PROSTHETIC HEART VALVES
}

Susana S. Meschengieser, MD ${ }^{a}$

Carlos G. Fondevila, MD

Juan Frontroth, $\mathrm{PhD}^{\mathrm{a}}$

María T. Santarelli, $\mathrm{MSc}^{\mathrm{b}}$

María A. Lazzari, MD
Background: Mechanical heart valve replacement requires lifelong anticoagulant treatment. Aspirin has proved useful in further reducing thromboembolic events when added to oral anticoagulants. However, increased (gastrointestinal) bleeding was observed at the doses previously tested for this combination in heart valve prostheses. Methods: We performed a prospective randomized trial to compare the combination of low-intensity oral anticoagulants (international normalized ratio 2.5 to 3.5 ) plus aspirin $(100 \mathrm{mg} / \mathrm{day})(\mathrm{arm} \mathrm{A})$ versus high-intensity oral anticoagulants alone (arm B) (international normalized ratio 3.5 to 4.5 ). Arm $A$ included 258 patients and arm B 245 patients. The two groups were comparable for all baseline characteristics. Results: The outcomes of the study were embolism, valve thrombosis, and major hemorrhage. The median follow-up was 23 months. The two treatments offered similar antithrombotic protection. The incidence of embolic episodes was 1.32 per 100 patient-years ( $95 \%$ confidence interval 0.53 to 2.7 ) for arm $A$ and 1.48 per 100 patient-years (95\% confidence interval 0.59 to 3.03) for arm B. Major hemorrhage occurred in 1.13 per 100 patient-years (95\% confidence interval 0.41 to 2.45 ) for arm $A$ and 2.33 per 100 patient-years (95\% confidence interval 1.17 to 4.14 ) for arm B. Gastrointestinal bleeding was not increased by this combined reduced dose of aspirin and coumarin. (J Thorac Cardiovasc Surg 1997; 113:910-6)
$T^{1}$ he past 20 years have witnessed dramatic improvements in the morbidity of prosthetic heart valves. Lifelong oral anticoagulation is recommended to reduce the incidence of systemic embolism. However, thromboembolism still arises in up to $2 \%$ of patients per year. ${ }^{1}$ Several trials tested the combination of an oral anticoagulant plus an anti-

From the Departamento de Hemostasia y Trombosis and Biostatistical Section, ${ }^{\mathrm{b}}$ Instituto de Investigaciones Hematólogicas "Mariano R. Castex," Academia Nacional de Medicina, Buenos Aires, Argentina.

Presented in abstracted form in the XIVth Congress of the International Society of Thrombosis and Haemostasis, New York, N.Y., July 1993.

Received for publication August 6, 1996; revisions requested Oct. 21, 1996; revisions received Nov. 19, 1996; accepted for publication Nov. 21, 1996.

Address for reprints: Susana S. Meschengieser, Departamento de Hemostasia y Trombosis, Instituto de Investigaciones Hematologicas "Mariano R. Castex," Academia Nacional de Medicina, Pacheco de Melo 3081, Buenos Aires, Argentina 1425.

Copyright (C) 1997 by Mosby-Year Book, Inc.

$0022-5223 / 97 \$ 5.00+0 \quad \mathbf{1 2 / 1 / 7 9 4 4 5}$ platelet agent in patients with prosthetic valves: the combined therapy tended to decrease the incidence and severity of embolic episodes with some increase in bleeding, mainly in the digestive tract. ${ }^{2-5}$ This was particularly true when aspirin at doses greater than $500 \mathrm{mg} /$ day was combined with high-intensity coumarin. The optimal therapeutic range for any antithrombotic scheme should be consistent with adequate thromboembolic protection but as low as possible to avoid therapy-related bleeding. Major bleeding events range between $0.7 \%$ to $6.3 \%$ per patient-year ${ }^{1,6}$ and the incidence increases as the international normalized ratio (INR) increases. ${ }^{7} \mathrm{~A}$ recent metaanalysis ${ }^{8}$ has compared the efficacy and safety of combined antiplatelet plus anticoagulant therapy with anticoagulant monotherapy: the combined therapy significantly reduced embolism by $67 \%$ along with a significant but undesirable $60 \%$ increment in hemorrhagic events. Furthermore, an impressive twofold increment in major gastrointestinal and other bleeding raised the question about a safer dose of aspirin, if any, to be combined with oral anticoagulants. 
Evidence indicates that gastrointestinal symptoms and bleeding associated with aspirin are dose dependent. ${ }^{9,}{ }^{10}$ Low doses of aspirin have demonstrated clear cardiovascular benefits, ${ }^{11}$ and the antithrombotic effects of aspirin were evident at doses greater than $100 \mathrm{mg} /$ day. A recent trial in patients with heart valve disease aimed at a lower aspirin dose $(100$ $\mathrm{mg} /$ day) while maintaining an INR between 3 and 4.5. ${ }^{12}$ However, the rates of bleeding showed that gastrointestinal and other major bleeding were still a problem for the aspirin group.

The efficacy and safety of the combination of low-dose aspirin plus a less intense oral anticoagulation has not been tested against standard-intensity oral anticoagulation. We performed a prospective randomized trial comparing oral anticoagulation (INR 2.5 to 3.5) in combination with $100 \mathrm{mg} / \mathrm{day}$ of plain aspirin versus oral anticoagulation alone (INR 3.5 to 4.5 ).

\section{Methods}

This study was opened for patient accrual in July 1988 and remained open through July 1992. The patients were followed up for a median of 23 months and for a maximum of 47 months. The study was open, because the investigators who saw the patients during the follow-up needed to adjust the acenocoumarol dosage to attain the targeted INR.

Patients with a mechanical prosthetic heart valve were randomized with a computer-generated program to arm A, oral anticoagulation (INR 2.5 to 3.5 ) plus aspirin (100 $\mathrm{mg} /$ day), or arm B, oral anticoagulation alone (INR 3.5 to 4.5).

Patients were randomized either promptly after the operation (when anticoagulation was considered to be adequate) or at different variable intervals between the operation and the start of this study, switching from a different therapy to one of the two arms of this protocol. Patients with previous gastrointestinal bleeding and patients with a previous history of embolic episodes or suspected hemorrhagic tendency were excluded from the study.

Follow-up endpoints included thromboembolism and major hemorrhage. Cerebral embolism was defined as a sudden neurologic event (focal motor weakness, visual deficit, or speech disturbance) with a computed tomographic brain scan negative for intracranial bleeding and for which there was no other clinical explanation. Embolic events were graded as to the presence or absence of any residual sequel, but transient ischemic events were also considered. Peripheral embolism was diagnosed by angiography or surgery. Valve thrombosis was confirmed by surgery. Major hemorrhage was considered present when the patient died or required transfusion or hospitalization. All other bleeding episodes were defined as minor.

Anticoagulation was monitored by prothrombin time $^{13,14}$ measured with the use of human brain thromboplastin with an international sensitivity index from 1.05 to 1.15 and a manual coagulation test. This was standardized using Argentine reference thromboplastin ${ }^{15}$ controlled versus international reference thromboplastin. ${ }^{16}$ The prothrombin time ratio was converted into international normalized ratio (INR).

All the patients enrolled in the study gave informed consent. Patients were repeatedly instructed to report any symptoms suggestive of embolism or hemorrhage. The percentage of anticoagulation controls within the aimed therapeutic range was calculated for each year of follow-up in each patient.

Statistical methods. To test whether the proportion of embolism was the same in the two groups at follow-up, we tested for equal proportions from two independent samples. ${ }^{17}$ The same was done for major hemorrhage and for the combined endpoint of embolism or major hemorrhage.

Time free of events was calculated from the entry date until the first occurrence of an embolism, a major hemorrhage, or embolism and/or major hemorrhage. For each of the endpoints, we used the product-limit method of Kaplan-Meier ${ }^{18}$ to plot the curve in each group on time free of the endpoint. The log-rank test ${ }^{19}$ was used to test whether these curves were equal.

The proportional hazards model developed by $\mathrm{Cox},{ }^{20}$ using the maximum partial likelihood ratio statistic, was used to assess the joint effect of treatment and patient characteristics (age, sex, valve design, valve position, postoperative cardiac rhythm, postoperative atrial size, interval between the operation and admission to the protocol, and concomitant coronary bypass procedure) on each of the three outcomes: embolism, major hemorrhage, and embolism and/or major hemorrhage.

All $p$ values are two-sided; a level of 0.05 was considered significant. Egret (SERO, Seattle, Wash.) and Stata (Stata Corporation, College Station, Tex.) were used to perform all the statistical analyses.

\section{Results}

Patient characteristics. A total of 503 patients were randomized into the study. The baseline characteristics are shown in Table I. The two groups were comparable except for the higher prevalence of atrial fibrillation in arm A. Fifty-three $(10.5 \%)$ of the 503 patients had concomitant surgical procedures, the most common being coronary artery bypass grafting in $21(8 \%)$ patients in arm $\mathrm{A}$ and 13 $(5 \%)$ patients in arm $\mathrm{B}$.

The total follow-up was 529 years for arm A and 471 years for arm B. The median follow-up was 23 months. Thirty patients (nine from arm A, 21 from arm B) died. One patient ( $\mathrm{arm} B$ ) died after a cerebral hemorrhage. Twenty-nine deaths were unrelated to the antithrombotic therapy. Two patients were withdrawn from the protocol because chronic renal failure developed and it was difficult to maintain their anticoagulation level; 20 patients from 
Table I. Baseline patient characteristics

\begin{tabular}{|c|c|c|c|c|}
\hline & \multicolumn{2}{|c|}{$\operatorname{Arm} A(n=258)$} & \multicolumn{2}{|c|}{$A m B(n=245)$} \\
\hline & No. & $\%$ & No. & $\%$ \\
\hline Median age (yr) & 54 & & 53 & \\
\hline Male patients & 154 & 60 & 138 & 56 \\
\hline Female patients & 104 & 40 & 107 & 44 \\
\hline \multicolumn{5}{|l|}{ Valve position } \\
\hline Aortic & 164 & 64 & 169 & 69 \\
\hline Mitral & 79 & 31 & 69 & 28 \\
\hline Tricuspid & 2 & 0.8 & 0 & \\
\hline Aortic and mitral & 13 & 5 & 7 & 3 \\
\hline \multicolumn{5}{|l|}{ Valve type } \\
\hline Starr-Edwards & 63 & 24 & 67 & 27 \\
\hline Disc $^{*}$ & 169 & 66 & 158 & 64 \\
\hline St. Jude Medical & 14 & 5 & 9 & 4 \\
\hline Unknown & 12 & 5 & 11 & 5 \\
\hline \multicolumn{5}{|l|}{ Cardiac rhythm } \\
\hline Atrial fibrillation & 53 & $20 \dagger$ & 36 & 15 \\
\hline Synus rhythm & 150 & 58 & 152 & 62 \\
\hline Pacemaker & 2 & 1 & 5 & 2 \\
\hline Unknown & 53 & 21 & 52 & 21 \\
\hline \multicolumn{5}{|l|}{ Associated surgical procedures } \\
\hline $\mathrm{CABG}$ & 21 & 75 & 13 & 52 \\
\hline Mitral commissurotomy & 3 & 11 & 8 & 32 \\
\hline Others & 4 & 14 & 4 & 16 \\
\hline \multicolumn{5}{|c|}{ Interval between surgery and admission to protocol } \\
\hline$<6 \mathrm{mo}$ & 128 & 50 & 121 & 49 \\
\hline$\geq 6 \mathrm{mo}$ & 130 & 50 & 124 & 51 \\
\hline
\end{tabular}

"Includes Björk-Shiley (Shiley, Inc., Irvine, Calif.), Medtronic Hall (Medtronic, Inc., Minneapolis, Minn.), Sorin (Sorin Biomedica Spa, Saluggio, Italy), and Omniscience (Medical Inc., Inver Grove Heights, Minn.).

$\dagger p=0.0438$.

Table II. Adequacy of anticoagulation

\begin{tabular}{lcccccc}
\hline & \multicolumn{3}{c}{ Arm A } & \multicolumn{3}{c}{ Arm B } \\
\cline { 2 - 6 } & Year 1 & Year 2 & Year 3 & Year 1 & Year 2 & Year 3 \\
\hline Mean INR & 3.05 & 3.18 & 3.1 & 3.78 & 4 & 4.06 \\
Adequate (\%) & 46 & 49 & 47 & 32 & 36 & 37 \\
Insufficient (\%) & 30 & 24 & 30 & 45 & 36 & 38 \\
Excessive (\%) & 24 & 27 & 23 & 23 & 28 & 25 \\
\hline
\end{tabular}

arm $\mathrm{A}(8 \%)$ and 20 patients from arm $\mathrm{B}(8 \%)$ were lost to follow-up.

Adequacy of anticoagulation. The median INR calculated for each year of evaluation was adequate in both arms of the protocol (Table II). Mean INRs for the first 3 years of follow-up were 3.11 in arm A and 3.98 in arm $\mathrm{B}(p=0.0000$ for the comparison of each of the years).

Thromboembolic episodes. The incidence of embolism was $2.7 \%$ (95\% confidence interval [CI] 1.09 to 5.5) for arm A and $2.8 \%$ (95\% CI 1.11 to 5.7) for arm B. The estimated survival free of embolism at 46 months was $94 \%$ (95\% CI 0.87 to 0.97 ) for arm A and $93 \%(95 \%$ CI 0.84 to 0.96$)$ for arm B $(p=$ $0.7008)$.

In arm $\mathrm{A}$ there were seven thromboembolic episodes (Table III). Total embolism rate was $2.7 \%$ or 1.32 episodes per 100 patient-years (95\% CI 0.53 to $2.7)$; for cerebral episodes only, the rate was $1.9 \%$ or 0.94 episodes per 100 patient-years $(95 \%$ CI 0.30 to 2.19). In arm $B$ there were seven thromboembolic events $(2.8 \%)$. One of the cerebral emboli underwent a hemorrhagic transformation. The annualized rate of embolism was of 1.48 episodes per 100 patient-years (95\% CI 0.59 to 3.03). Prothrombin times at the time of the embolism were available in 
Table III. Thromboembolic episodes

\begin{tabular}{|c|c|c|c|c|}
\hline & \multicolumn{2}{|c|}{$\operatorname{Arm} A(n=258)$} & \multicolumn{2}{|c|}{$A m{ }^{\prime} B(n=245)$} \\
\hline & No. & $\%$ & No. & $\%$ \\
\hline Cerebral with sequelae & 1 & 14 & 3 & 43 \\
\hline Cerebral without sequelae (TIA) & 4 & 57 & 4 & 57 \\
\hline Valve thrombosis & 1 & 14 & & \\
\hline Peripheric & 1 & 14 & & \\
\hline Rate & 7 & 2.7 & 7 & 2.8 \\
\hline Episodes per 100 patient-years & \multicolumn{2}{|c|}{$\begin{array}{l}1.32 \\
\quad(95 \% \text { CI } 0.53 \text { to } 2.7)\end{array}$} & \multicolumn{2}{|c|}{$\begin{array}{l}1.48 \\
(95 \% \text { CI } 0.59 \text { to } 3.03)\end{array}$} \\
\hline
\end{tabular}

TLA, Transient ischemic attack.

six of the seven patients in arm A: in five of the six patients $(83 \%)$ the prothrombin times were within the therapeutic range, and in one $(17 \%)$ anticoagulation was inadequate. In arm B, three of seven patients had prothrombin times $(43 \%)$ within the therapeutic range, three $(43 \%)$ had inadequate anticoagulation, and one (14\%) had excessive anticoagulation. Two of the emboli in each group arose in patients who had atrial fibrillation.

Position of the valve. Of the seven patients having embolic events in arm A, four (57\%) had mitral prostheses and three had aortic valves (43\%). In arm B, two (29\%) of the seven patients having embolic episodes had mitral prostheses and five (71\%) had aortic valves. The rate of embolism for mitral and aortic valves in arm A was 5.06 or 2.5 ( $95 \%$ CI 0.68 to 6.28 ) episodes per 100 patient-years and 1.83 or 0.87 ( $95 \%$ CI 0.18 to 2.54 ) episodes per 100 patient-years, respectively. In arm B the rate of embolism for mitral and aortic valves was 2.82 or 1.44 (95\% CI 0.17 to 5.10) episodes per 100 patientyears and 2.99 or 1.59 (95\% CI 0.52 to 3.69 ) episodes per 100 patient-years, respectively. The estimated survival free of embolism at 46 months was $94 \%$ (95\% CI 0.86 to 0.97 ) for mitral valves, 93\% (95\% CI 0.86 to 0.93 ) for aortic valves, and $100 \%$ for double prostheses $(p=0.1321)$.

Type of valve. The rates of embolism were 6.34 (95\% CI 1.75 to 15.4 ) and $1.56(95 \%$ CI 0.32 to 4.49$)$ $(p=0.0842)$ for caged-ball versus non-caged-ball valves for arm $\mathrm{A}$ and 7.67 (95\% CI 2.46 to 16.66) and 1.13 (95\% CI 0.13 to 4.04$)(p=0.0183)$ for arm $\mathrm{B}$, respectively.

Bleeding complications. The incidence of major bleeding was $2.32 \%$ (95\% CI 0.85 to 4.99 ) for arm A and $4.49 \%$ (95\% CI 2.26 to 7.89 ) for arm B. The estimated survival free of hemorrhage at 46 months was $97 \%(95 \% \mathrm{Cl} 0.92$ to 0.99$)$ for arm A and $91 \%$ ( $95 \%$ CI 0.84 to 0.95 ) for arm B $(p=0.1064)$.
Table IV shows the sites of major and minor bleeding episodes. There were six $(2.32 \%)$ major bleeding episodes in arm A (1.13 per 100 patientyears; $95 \%$ CI 0.41 to 2.45 ), and $11(4.49 \%)$ in arm B (2.33 per 100 patient-years; $95 \%$ CI 1.17 to 4.14 ). This represents a reduction in the relative risk of major bleeding for the less intense anticoagulation arm of $52 \%$ ( $95 \%$ CI 0.19 to 1.38$)(p=0.2731)$. Three cerebral hemorrhages occurred in arm B and one was fatal. The incidence of major gastrointestinal bleeding was higher in arm B (2.45\% or 1.27 per 100 patient-years; $95 \%$ CI 0.46 to 2.75 ) than in arm A $(0.77 \%$ or 0.37 per 100 patient years; $95 \%$ CI 0.04 to 1.35$)$.

In $\operatorname{arm} \mathrm{A}$, a duodenal ulcer was identified as a predisposing factor in one of three patients with gastrointestinal bleeding; in one patient with gynecologic bleeding, a previous bleeding tendency was identified. In arm B, five of the 11 patients with major bleeding had possible predisposing factors: recent cranial trauma, lung carcinoma, vascular malformation in three patients with cerebral bleeding, chronic renal failure, and bacterial endocarditis in two patients with gastrointestinal bleeding. Even after the exclusion of patients with a predisposing cause, the rate of gastrointestinal bleeding was more than double in the arm with more intense anticoagulation without aspirin (Table IV).

Four $(66 \%)$ of the six patients with hemorrhagic complications in arm A had slightly excessive anticoagulation, one (17\%) had adequate anticoagulation, and the remaining patient $(17 \%)$ had insufficient anticoagulation. In arm B, three $(27 \%)$ of the 11 with hemorrhagic complications had excessive anticoagulation (one with a fatal cerebral hemorrhage) and five (45\%) had insufficient anticoagulation.

Failure-free survival. Thromboembolism or major hemorrhage occurred in 13 patients in arm A 
Table IV. Bleeding episodes

\begin{tabular}{|c|c|c|c|c|}
\hline & \multicolumn{2}{|c|}{$\operatorname{Arm} A(n=258)$} & \multicolumn{2}{|c|}{$\operatorname{Arm} B(n=245)$} \\
\hline & No. & $\%$ & No. & $\%$ \\
\hline \multicolumn{5}{|l|}{ Major bleeding } \\
\hline Intracranial & & & $3^{*}$ & 27 \\
\hline Gastrointestinal $\dagger$ & 3 & 50 & 6 & 54 \\
\hline Menorrhagia & 3 & 50 & 1 & 9 \\
\hline Soft tissue hematoma & & & 1 & 9 \\
\hline Rate & 6 & $2.3 \%$ & 11 & 4.5 \\
\hline Episodes per 100 patient-years & \multicolumn{2}{|c|}{$\begin{array}{l}1.13 \\
(95 \% \mathrm{CI} 0.41 \text { to } 2.45)\end{array}$} & \multicolumn{2}{|c|}{$\begin{array}{l}2.33 \\
(95 \% \text { CI } 1.17 \text { to } 4.14)\end{array}$} \\
\hline \multicolumn{5}{|l|}{ Minor bleeding } \\
\hline Hematuria & 12 & 34 & 6 & 15 \\
\hline Epistaxis & 10 & 28 & 8 & 19 \\
\hline Gastrointestinal $\uparrow$ & 2 & 6 & 6 & 15 \\
\hline Menorrhagia & 4 & 11 & 3 & 7 \\
\hline Ecchymoses & 1 & 2.8 & 8 & 19 \\
\hline Hemoptysis & & & 2 & 5 \\
\hline Gingival bleeding & & & 2 & 5 \\
\hline Proctorrhagia & 1 & 2.8 & 5 & 12 \\
\hline Others & 5 & 14 & 1 & 2.4 \\
\hline Rate & 35 & 14 & 41 & 17 \\
\hline
\end{tabular}

\footnotetext{
*One lethal.
}

$\dagger$ The total number of gastrointestinal hemorrhages after the exclusion of patients with a potential predisposing cause was 4 or 0.76 per 100 patient-years ( $95 \%$ CI 0.20 to 1.92 ) and 10 or 2.12 per 100 patient-years $(95 \%$ CI 1.02 to 3.86$)$ for arms $\mathrm{A}$ and $\mathrm{B}$, respectively $(p=0.1425)$.

(5.03\% or 2.45 per 100 patient-years; $95 \%$ CI 1.31 to 4.16) and 18 patients in arm B (7.34\% or 3.82 per 100 patient-years; 95\% CI 2.28 to 5.97 ). The actuarial risk of embolism and major bleeding at 40 months (Fig. 1) was $10 \%$ for arm A and $15 \%$ for arm. $\mathrm{B}(p=0.1576)$. This represents a relative risk reduction of $69 \%(95 \% \mathrm{CI} 0.34$ to 1.37$)(p=$ 0.3732).

Cox multivariate analysis. Several Cox models were performed, using treatment and the set of patient characteristics at diagnosis, that will lead to an adjusted effect of treatment on outcomes.

One set of models was developed for embolism, in which the presence of postoperative atrial size $(>50$ $\mathrm{mm}$ ) was a statistically significant predictor of embolism.

Finally, a Cox model was developed for the joint effect of embolism and major hemorrhage, in which postoperative atrial size was the only significant predictor of events.

\section{Discussion}

In this randomized trial, we demonstrated that the addition of $100 \mathrm{mg}$ daily aspirin to a less intense oral anticoagulation program offered the same antithrombotic protection and did not increase the risk of bleeding than a more intense anticoagulant-alone regimen. Moreover, a better quality of life (event- free survival) and a tendency toward fewer and milder episodes of cerebral embolism were offered by the combined treatment. Although embolism was more prevalent in caged-ball valves in both groups, the difference reached statistical significance only in the anticoagulant-alone group.

When this trial was designed, the currently suggested practice for patients with prosthetic heart valves was to prolong the INR from 3.0 to $4.5 .^{21}$ The last consensus on oral anticoagulation ${ }^{22}$ suggested a lower INR of 2.5 to 3.5 for patients with non-cagedball prostheses. This recommendation was substantiated in a literature review. ${ }^{23}$ However, there are no randomized clinical trials comparing the old ( 3 to 4.5 ) versus the newer (2.5 to 3.5 ) recommended intensities. Moreover, a substantial number of patients with old, more thrombogenic prostheses still attend the anticoagulation clinics. Whether the same level of anticoagulation is required when coumarin is combined with a platelet inhibitor is unknown. The addition of aspirin to oral anticoagulation showed a significative reduction in the rate of thromboembolism., 3 However, the incidence of gastrointestinal bleeding was increased when aspirin was given at dosages of $1 \mathrm{gm} / \mathrm{day}^{2}$ or $250 \mathrm{mg}$ twice daily. ${ }^{3}$ Despite the excessive bleeding, the thromboembolic protection was sometimes poor. ${ }^{4}$ In a recent study by Altman and associates, ${ }^{5}$ lower intensity 


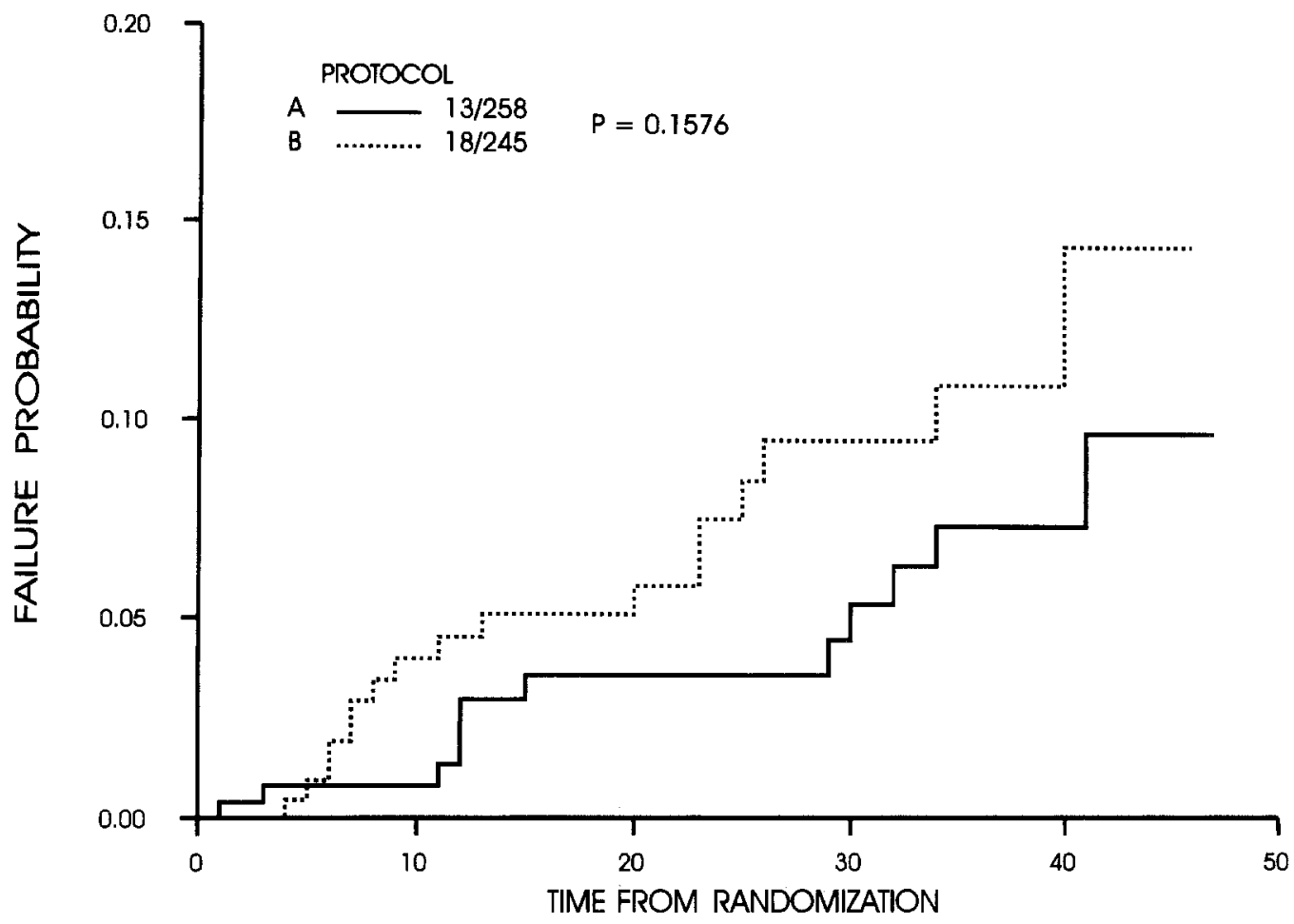

Fig. 1. Efficacy and safety. Cumulative risk of events (embolism and/or major hemorrhage) attributable to antithrombotic therapy.

anticoagulation was as effective as and safer than a higher intensity regimen, at least when combined with two platelet inhibitors. The lack of a control group treated with warfarin alone and the fact that most of the hemorrhages occurred within the targeted level of anticoagulation raises the question about the minimal (but still effective) dosage of aspirin.

If the deleterious effect of aspirin is a function of the dose, ${ }^{9}$ one can speculate whether a lower dose could retain efficacy while reducing the bleeding. Recently, low-dose aspirin has proven successful in lowering cardiac and cerebral vascular events. Turpie and coworkers ${ }^{12}$ found that $100 \mathrm{mg}$ aspirin added to warfarin (INR 3 to 4.5 ) reduced significantly the embolic rates, when compared with a warfarin-alone regimen of similar intensity. However, the mean INRs for both aspirin and placebo groups were similarly low (3.1 and 3 ), and $49 \%$ of the INR measurements fell below the targeted level. Thus it might be feasible that such low levels of anticoagulation were not optimum to prevent embolism in the warfarin-alone group, raising uncertainties about the performance of the combined therapy if tested against a more intense, better performing anticoagulant-alone regimen. The safety of the association might be questioned, because more total, major, and gastrointestinal hemorrhages occurred among patients treated with warfarin plus aspirin. ${ }^{12}$ A recent metaanalysis that confirmed the benefits of adding aspirin to coumarin in further reducing thromboembolism revealed an impressive increment in iatrogenic bleeding, which makes unacceptable the combination of doses of aspirin greater than $100 \mathrm{mg}$ or intensities greater than INR 3.5. ${ }^{8}$ Recent trials aimed at a less intense anticoagulant schedule: INR below 3.5..$^{24,25}$ An adequate embolism protection and a reduced risk of hemorrhagic complications was found. Unfortunately, these trials did not include standard (intense) anticoagulation or combined anticoagulant-plus-aspirin groups. Moreover, in Turpie's trial the warfarinalone arm (mean INR 3.1) showed a higher (4.6 per 100 patient-years) incidence of embolism. Hence evidence about the antithrombotic efficacy of lowintensity anticoagulation alone is conflicting, and it is not clear whether results from these less intense regimens can be improved by adding aspirin or other antiplatelet agents.

In our study we had a low incidence of systemic embolism in both arms (1.32 and 1.48 per 100 patient- 
years). Most of our episodes were cerebral (five of seven in arm A and seven of seven in arm B) and left no neurologic deficit in four of five and four of seven patients. Despite the similarly low incidence of embolism, the aspirin arm showed a tendency to more benign (transient) cerebral episodes. Furthermore, patients having caged-ball valves benefited from the combined therapy. This benefit was not counterbalanced by an increase in bleeding: we found the rate of major bleeding episodes in the less intense arm (1.13 and 2.33 per 100 patient-years, respectively) dropped by more than half. Gastrointestinal bleeding was not increased by the addition of aspirin.

Our results indicate that the addition of low-dose aspirin to a low-intensity oral anticoagulant regimen in this selected population free from gastrointestinal hemorrhage or hemorrhagic history conferred antithrombotic protection as effectively as the highintensity "standard" anticoagulation without increasing the likelihood of major hemorrhages. The effects were not limited to any particular subgroup of patients with mechanical valves. Further trials seem warranted, looking for special subgroups (elderly patients or those with coronary or cerebrovascular disease) that could be particularly benefited by the combination therapy.

\section{REFERENCES}

1. Cannegieter SC, Rosendaal FR, Briet E. Thromboembolic and bleeding complications in patients with mechanical heart valve prostheses. Circulation 1994;89:635-41.

2. Dale J, Myhre E, Storstein O, Stormorken H, Efskind L. Prevention of arterial thromboembolism with acetylsalicylic acid: a controlled clinical study in patients with aortic ball valves. Am Heart J 1977;94:101-11.

3. Altman R, Boullon F, Rouvier J, Raca R, De La Fuente L, Favaloro R. Aspirin and prophylaxis of thromboembolic complications in patients with substitute heart valves. J Thorac Cardiovasc Surg 1976;72:127-9.

4. Chesebro JH, Fuster V, Elveback LR, McGoon DC, Pluth JR, Puga FJ, et al. Trial of combined warfarin plus dipyridamole or aspirin therapy in prosthetic heart valve replacement: danger of aspirin compared with dipyridamole. Am J Cardiol 1983;51:1537-41.

5. Altman R, Rouvier J, Gurfinkel E, D’Ortencio O, Manzanel R, De La Fuente L, Favaloro RG. Comparison of two levels of anticoagulant therapy in patients with substitute heart valves. J Thorac Cardiovasc Surg 1991;101:427-31.

6. Stein PD, Alpert JS, Copeland J, Dalen JE, Goldman S, Turpie AGG. Antithrombotic therapy in patients with mechanical and biological prosthetic heart valves. Chest 1992; 102:445S-55S.

7. Wilson DB, Dunn MI, Hassanein K. Low intensity anticoagulation in mechanical cardiac prosthetic valves. Chest 1991; 100:1553-7.

8. Cappelleri JC, Fiore LD, Brophy MT, Deykin D, Lau J. Efficacy and safety of combined anticoagulant and antiplatelet therapy versus anticoagulant monotherapy after heart-valve replacement: a meta-analysis. Am Heart J 1995;130:547-52.

9. Hawthorne AB, Mihada YR, Cole AT, Hawkey J. Aspirin induced gastric mucosal damage: prevention by enteric coating and relation to prostaglandin synthesis. $\mathrm{Br} \mathrm{J}$ Clin Pharmacol 1991;32:77-83.

10. Hirsh J, Salzman EW, Harker L, Fuster V, Dalen JE, Cairns JA, et al. Aspirin and other antiplatelet active drugs: relationship among dose, effectiveness and side effects. Chest 1989;95:512-8.

11. ISIS 2 (Second International Study of Infarct Survival Collaborative Group. Randomized trial of intravenous streptokinase, oral aspirin, both or neither among 17,187 cases of suspected acute myocardial infarction. Lancet 1988;2:349-60.

12. Turpie AGG, Gent M, Laupacis A, Latour Y, Gunstensen J, Basile F, et al. A comparison of aspirin with placebo in patients treated with warfarin after heart-valve replacement. N Engl J Med 1993;329:524-9.

13. Quick AJ. The prothrombin time in haemophilia and in obstructive jaundice. J Biol Chem 1935;109:73-4.

14. Biggs R, Denson KVE. Standardization of the one-stage prothrombin time for control of anticoagulant therapy. Br $\mathrm{J}$ Med 1967;1:84-8.

15. Araldi HT, Martínez Canaveri A, Albertengo ME, Cinto RO, Elgue G. Primer Reactivo Nacional Argentino de Referencia de Tromboplastina de cerebro humano. Sangre 1980;25:421-4.

16. International Committee for Standardization in Haematology, International Committee on Thrombosis and Haemostasis. ICSH/ICTH recommendations for reporting prothrombin time in oral anticoagulant control. Thromb Haemost 1985;53:155-6.

17. Fleiss JL. Statistical methods for rates and proportions, 2nd ed. New York: John Wiley, 1981:14-5.

18. Kaplan EL, Meier P. Nonparametric estimation for incomplete observations. J Am Stat Assoc 1958;53:457-81.

19. Peto R, Pike MC, Armitage P, Breslow NE, Cox DR, Howard SV, et al. Design and analysis of randomized clinical trials requiring prolonged observation of each patient. II. Analysis and examples. Br J Cancer 1978;35:1-35.

20. Cox DR. Regression models and life-tables. J R Stat Soc 1972:34:187-220.

21. Stein PD, Kantrowitz A. Antithrombotic therapy in mechanical and biological prosthetic heart valves and saphenous vein bypass grafts. Chest 1989;95(Suppl):107S-17S.

22. Stein PD, Alpert JS, Copeland J, Dalen JE, Goldman S, Turpie AGG. Antithrombotic therapy in patients with mechanical and biological prosthetic heart valves. Chest 1995; $108: 371 \mathrm{~s}-9 \mathrm{~s}$.

23. Stein PD, Grandison D, Hua TA, Slettehaugh PM, Henry JW, Turlapaty P, et al. Therapeutic level of oral anticoagulation with warfarin in patients with mechanical prosthetic heart valves: review of literature and recommendations based on international normalized ratio. Postgrad Med J 1994(Suppl I):S72-83.

24. Butchart EG, Lewis PA, Grunkemeier LA, Kulatilake N, Breckenridge IM. Low risk of thrombosis and serious embolic events despite low-intensity anticoagulation: experience with 1,004 Medtronic Hall valves. Circulation 1988;78(Suppl):I66-77.

25. Maragos K, Spiliotopoulou I, Digenopoulou L, Sevastos N, Theodossiades G, Kontopoulou-Griva I. Less intensive antithrombotic therapy in patients with mechanical prosthetic heart valves. Thromb Haemostas 1993;69:676. 